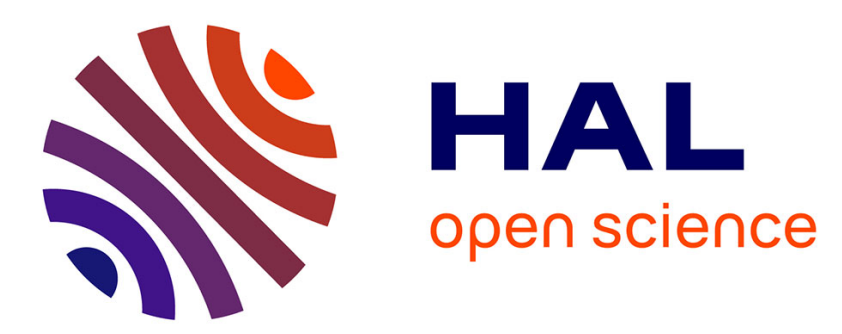

\title{
Aerosol size distribution measurement using a SMPS: scanning mode and uncertainty analysis
}

Loic Coquelin, Nicolas Fischer, Laurent Le Brusquet, François Gensdarmes

\section{To cite this version:}

Loic Coquelin, Nicolas Fischer, Laurent Le Brusquet, François Gensdarmes. Aerosol size distribution measurement using a SMPS: scanning mode and uncertainty analysis. I2MTC 2012, May 2012, Graz, Austria. pp.1718-1721, 10.1109/I2MTC.2012.6229546 . hal-00736539

\section{HAL Id: hal-00736539 \\ https://hal-centralesupelec.archives-ouvertes.fr/hal-00736539}

Submitted on 28 Sep 2012

HAL is a multi-disciplinary open access archive for the deposit and dissemination of scientific research documents, whether they are published or not. The documents may come from teaching and research institutions in France or abroad, or from public or private research centers.
L'archive ouverte pluridisciplinaire HAL, est destinée au dépôt et à la diffusion de documents scientifiques de niveau recherche, publiés ou non, émanant des établissements d'enseignement et de recherche français ou étrangers, des laboratoires publics ou privés. 


\title{
Aerosol size distribution measurement using a SMPS: scanning mode and uncertainty analysis
}

\author{
Coquelin L., Fischer N., Le Brusquet L., Fleury G., Motzkus C., Gensdarmes F.
}

\begin{abstract}
A model to simulate SMPS (Scanning Mobility Particle Sizer) measurement and the associated uncertainty analysis when axial DMA (Differential Mobility Analyser) classifier operates under scanning mode conditions is described. Starting from simulated SMPS raw data, a fast estimation of aerosol size distribution measurement using regularization technique is performed. Then, global sensitivity analysis is used to discriminate significant parameters of the system and, as a preliminary result, a $95 \%$ confidence region is obtained by Monte Carlo simulations on an atmospheric aerosol size distribution.
\end{abstract}

Index Terms-Aerosol Size distribution measurement, Electrical classification, uncertainty analysis, Data inversion

\section{INTRODUCTION}

A MONG devices using electrical mobility methods to measure aerosol particle size distribution, the SMPS (Scanning Mobility Particle Sizer) is the most widely used to characterize particles smaller than $0.1 \mu \mathrm{m}$. Since it can be applied in fields like: indoors air quality measurement, vehicle exhaust pipes, atmospheric studies, toxicology testing, etc., it has become of interest to assess the uncertainty associated with its outputs. So far, although some works have been carried on the adequacy of particle size measurements made by this device, such a dimensional feature uncertainty cannot be provided. First, a full SMPS model is presented when DMA operates under scanning mode. Then, an alternative to common data inversion performed in aerosol science that allies fast reconstruction of the aerosol size distribution and accuracy is described. Fast reconstruction of the solution is a requirement here due to the fact that when dealing with uncertainty propagation in a numerical code, a large number of calls is necessary. Last section pays a particular attention to uncertainty analysis and Monte-Carlo simulations lead to a $95 \%$ confidence region on an atmospheric aerosol size distribution. Figure 1 illustrates how to perform one simulation of the numerical code: starting from a known aerosol size distribution $n\left(d_{p}\right), d_{p} \in\left[d_{\min }, d_{\max }\right]$ being the interval of scanned diameters, the code returns an estimate $\hat{n}\left(d_{p}\right)$.

\section{SCANNING MODE THEORY}

SMPS is composed of a Differential Mobility Analyser (DMA) and a Condensation Particle Counter (CPC). First one selects particles mobility diameters while the other is an optical counter used for the detection phase. First, aerosol passes through an inertial impactor to avoid largest particles to enter the DMA column, then the aerosol enters the charge neutraliser to be conditioned. Indeed, aerosol particles are usually charged. TSI neutraliser is used here to create a bipolar charge distribution by exposing aerosol

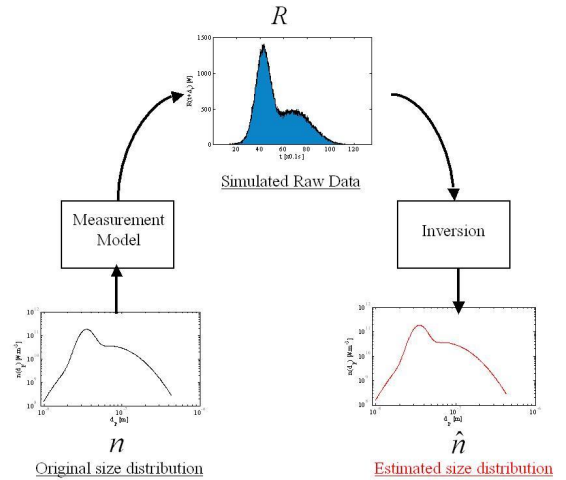

Fig. 1. Scheme of the simulation principle

to a radioactive Krypton source, so, particles that carry several charges lose their charge excess. Once aerosol is well conditioned particles are selected using electrical classification inside DMA column: an electric field is created, and the airborne particles drift in the DMA according to their electrical mobility.

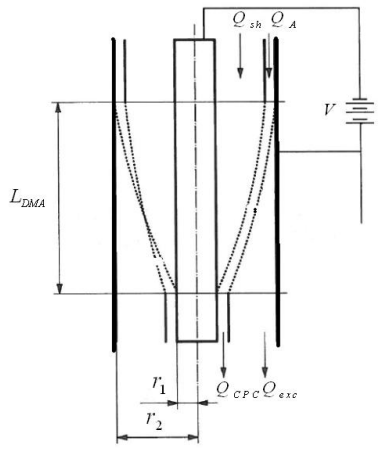

Fig. 2. Schematic diagram of a cylindrical DMA column

Flow rates system inside the DMA column and also geometrical parameters are described in the figure 2. $L_{D M A}$, $r_{1}$ and $r_{2}$ represent the length of the column, radii of the inner and outer electrodes, respectively. $Q_{s h}$ is the clean sheath air flow, $Q_{a}$ being the aerosol flow, $Q_{c p c}$ the mono disperse flow that goes to the aerosol detector and finally $Q_{\text {exc }}$ the excess flow.

Once selected, particles reach the CPC to be counted. Scanning mode is based on Wang and Flagan research [1]: voltage at the center rod of the DMA is ramped exponentially to ensure particles trajectories to remain unchanged during the all scanning time. Let $t_{s}$ the scanning time, $V_{\min }$ and $V_{\max }$ respectively the minimum and maximum 
value of the voltage defined by the user, Collins and al. [2] give the following expression for the tension:

$$
V(t)=V_{\min } \exp (t / \tau), \text { with } \tau=t_{s} / \ln \left(V_{\min } / V_{\max }\right) .
$$

CPC detects particles carried by flow rate $Q_{c p c}$ during time $\Delta_{t}$. Usually, CPC results are expressed in terms of concentration. Choice is made here to work with CPC raw data. Let $d_{p}$ be the particle mobility diameter, $Z_{d}$ the corresponding electrical mobility, $p$ the number of charges carried by each particle, $\Delta \phi(V)$ a function of the geometry and the supplied voltage of the DMA and $n\left(d_{p}\right)$ the aerosol size distribution, then basic equation for electrical mobility measurement leads to the $\mathrm{CPC}$ raw data response:

$$
r\left(t+\Delta_{t}\right)=\int_{0}^{+\infty} k\left(d_{p}, Z_{d}\left(p, d_{p}\right), \Delta \phi(\overline{V(t)})\right) n\left(d_{p}\right) d_{d_{p}}(1)
$$

where $r\left(t+\Delta_{t}\right)$ is the number of particles counted by the $\mathrm{CPC}$ at time $t+\Delta_{t}$ for particles selected in the DMA column set at voltage $\overline{V(t)}$ that is the tension of selection defined as the mean tension for a passage inside the column of the classifier:

$$
\overline{V(t)}=\frac{1}{t_{f}} \int_{t-t_{d}-t_{f}}^{t-t_{d}} V(\xi) d_{\xi} .
$$

NB: Particles counted by the CPC at time $t+\Delta_{t}$ have entered the DMA at time $t-t_{d}-t_{f}$ and have left it at time $t-t_{d}$. So, valid counts start from $t>t_{d}+t_{f}$ and end at $t>t_{s}+t_{d}$.

Indeed, particles reach the CPC only after transport through the DMA and transport through the tubing which connects the $\mathrm{CPC}$ with the mono disperse outlet port of the DMA. Transport times are respectively called $t_{f}$ and $t_{d}$. First is assumed to be equal to the mean residence time of the aerosol inside the DMA column and second depends on the internal CPC delay time and flow rates system (default value is for 10 inches connection to CPC in our case), so

$$
t_{f}=\frac{\pi\left(r_{2}^{2}-r_{1}^{2}\right) L_{D M A}}{Q_{s h}+Q_{a}},
$$

DMA and CPC are respectively characterized by the transfer function $\Omega$ and size detection efficiency $n_{C P C}$ when charge conditioner is modelled with function $\Psi$. For a given time $t$, the kernel function $k$ is given by the following expression:

$$
\begin{aligned}
& k\left(d_{p}, Z_{d}\left(p, d_{p}\right), \Delta \phi \overline{V(t)}\right) \\
& =Q_{a} \Delta_{t} \sum_{p=1}^{+\infty} \Psi\left(p, d_{p}\right) n_{C P C}\left(d_{p}\right) \Omega\left(Z_{d}\left(p, d_{p}\right), \Delta \phi \overline{V(t)}\right) .
\end{aligned}
$$

Charge conditioning model is extracted from both Wiedensohler [3] and Gunn [4] while $n_{C P C}$ is obtained by fitting experimental data made by Quant [5] by using cubic interpolation.

Next, modelling DMA transfer function requires the electrical mobility to be known for each mobility diameter $d_{p}$. Let $e$ the charge of the electron, $\mu_{g}$ the dynamic gas viscosity, $C_{c}$ the slip correction factor, electrical mobility is then linked to a specific mobility diameter $d_{p}$ via:

$$
Z_{d}\left(p, d_{p}\right)=\left(p e C_{c}\left(d_{p}\right)\right) /\left(3 \pi \mu_{g} d_{p}\right),
$$

where $C_{c}$ is defined as

$$
\begin{aligned}
C_{c}\left(d_{p}\right) & =1+K_{n}\left(d_{p}\right)\left[\alpha+\beta \exp \left(-\gamma / K_{n}\left(d_{p}\right)\right)\right], \\
K_{n}\left(d_{p}\right) & =\left(2 \lambda_{m}\right) / d_{p},
\end{aligned}
$$

where $K_{n}$ is the Knudsen number and $\lambda_{m}$ is the mean free path of a particle. Triplet $(\alpha, \beta, \gamma)$ is taken from experiments made by Kim and al. [6].

An other expression of the electrical mobility, denoted by $Z_{v}$ is:

$$
Z_{v}=\nu_{r} / E
$$

$\nu_{r}$ being the drift velocity and $E$ the electric field strength. Starting from equation (3), solving electromagnetic wave equation while assuming: quasi stationary system, uniformity of the velocity profiles inside the DMA column, and laminar flow regime instantaneously reached, one can get the electrical mobility $Z_{v}$ for a set voltage $V$ at the center rod voltage of the DMA:

$$
Z_{v}(V)=\frac{Q}{4 \pi \Delta \phi(V)}, \text { with } \Delta \phi(V)=\frac{L_{D M A} V}{\ln \left(r_{2} / r_{1}\right)} .
$$

From both expressions (2) and (4), knowing the voltage at which the particle has been selected, one can get its corresponding mobility diameter. Neglecting diffusional effects inside the column of the classifier, Whitby [7] showed the following expression for the DMA transfer function:

$$
\begin{aligned}
\Omega\left(Z_{d}, \Delta \phi(\overline{V(t)})\right) & \\
= & \frac{1}{Q_{a}} \max \left(0,\left[\operatorname { m i n } \left(Q_{a}, Q_{c p c},\left[\left(Q_{a}+Q_{c p c}\right) / 2-\mid 2 \pi\right.\right.\right.\right. \\
& \left.\left.\left.\left.Z_{d} \Delta \phi(\overline{V(t)})+\left(Q_{s h}+Q_{e x c}\right) / 2 \mid\right]\right)\right]\right) .
\end{aligned}
$$

Until there, a deterministic model that takes into account the physical considerations of the system has been built. The physic is here represented through analytical functions, so the need of having a code that runs quickly is fulfilled.

\section{DATA INVERSION}

Reconstruction of an unknown aerosol size distribution is a general mathematical problem: starting from CPC measurements, problem consists in the estimation of original concentration $n$. Through years several techniques have been found to reach this purpose [8], [9], [10], etc.. Nevertheless, industrial approaches have to fulfill other constraints such as a fast reconstruction of the signal. Main problem here is to reach enough accuracy in a short time.

\section{A. Common approach}

Since Spectrometers operate at high resolution, at each time of the scanning process, the support of $\Omega$ is narrow and it is common to consider that only a particle of diameter $d_{p}^{*}(t)$ is extracted from the classifier. When assuming 
that particles only carry one charge, this diameter is computed by solving the following equation:

$$
Z_{d}\left(p=1, d_{p}^{*}(t)\right)=Z_{v}(\overline{V(t)}),
$$

and if $\hat{n}$ denotes the estimate of the aerosol size distribution, its expression takes the following form:

$$
\hat{n}\left(d_{p}^{*}(t)\right)=\frac{r\left(t+\Delta_{t}\right)\left(\partial Z_{d}\left(1, d_{p}\right) / \partial d_{p}\right)_{d_{p}=d_{p}^{*}(t)}}{Q_{a} \Delta_{t} \Psi\left(1, d_{p}^{*}(t)\right) n_{C P C}\left(d_{p}^{*}(t)\right) \int_{Z_{v}^{-}}^{Z_{v}^{+}} \Omega d_{Z_{d}}},
$$

where

$Z_{v}^{ \pm}=Z_{v}(\overline{V(t)}) \pm \Delta Z_{v}(\overline{V(t)})=\left(1 \pm \frac{Q_{a}+Q_{c p c}}{Q_{s h}+Q_{e x c}}\right) Z(\overline{V(t)})$.

Multiple charge correction is used afterwards to estimate aerosol size distribution. Indeed, since electrical mobility function described in (2) is not bijective: $\exists\left(p^{\prime}, d_{p}^{\prime}\right)$ such that $Z_{d}\left(p^{\prime}, d_{p}^{\prime}\right)=Z_{d}\left(p, d_{p}\right)$, large particles carrying multiple charges have the same mobility as smaller particles carrying less charges. $n\left(d_{p}\right)$ is to describe aerosol particles carrying only one charge, so is the necessity of the correction.

\section{B. Alternative approach}

Estimation of the aerosol size distribution can be performed regardless of the DMA resolution being used and without assumptions concerning the number of charges carried by each particle. The method proposed here is based on the discretization of the mobility diameter space. It is somehow comparable to what has been developed by Alofs and Hagen [9] when the DMA operates under stepping mode.

First step is to discretize the mobility diameter space using log scale. Let $\left(d_{p, j}\right)_{j=1, \ldots, J}$ the points of reconstruction of the solution, because the particles diameters typically range over several orders of magnitude, a convenient choice for integrating (1) is the variable transformation $f\left(d_{p}\right)=\ln \left(d_{p}\right)$. Second step is to foresee the behaviour of the size distribution over a narrow diameter range. Linear interpolation is chosen here because changes in the solution are slow. Moreover, the more points is used to interpolate, the less the assumption will be strong. When using notations $f=f\left(d_{p}\right)$ and $f_{j}=f\left(d_{p, j}\right)$, equation (1) is further converted to:

$$
r\left(t+\Delta_{t}\right)=\int_{f_{1}}^{f_{J}} d_{p} k\left(f, Z_{d}(p, f), \Delta \phi \overline{V(t)}\right) n(f) d_{f},
$$

and it can be written in a matrix form as $R=K N$, where both solution vector $N$ and vector of the observations $R$ are defined as

$$
\begin{array}{ll}
R_{i}=r\left(t_{i}\right) & , t_{i}=t_{0}+i \Delta_{t}, \\
N_{j}=n\left(f_{j}\right) & , j=1, \ldots, J,
\end{array}
$$

while element $(i, j)$ of matrix $K$ corresponds to the following sum of integrals:

$$
\int_{f_{j-1}}^{f_{j}} d_{p} k(f) L_{j-1}(f) d_{f}+\int_{f_{j}}^{f_{j+1}} d_{p} k(f)\left(1-L_{j}(f)\right) d_{f},
$$

where $L_{j}$ is the linear interpolant. Good point of this method is that multiple charges correction won't have to be performed any longer because it is corrected by construction.

Knowing $R$ and $K$ expressions, problem now is to retrieve $N$. Nevertheless, $K$ is badly conditioned. Regularisation technique described by Tikkhonov [11] is used to overpass this problem. So, if typical least-square method leads to the minimization of the quadratic error $\| K N-$ $R \|^{2}$, Tikkhonov suggests to minimize $\|K N-R\|^{2}+\lambda\|N\|^{2}$. A prior on the solution to rebuild can be carried by the second term multiplied by regularisation parameter $\lambda$. Another prior is the positivity of the searched vector $N$ as it represents a concentration. As decided by Seinfeld [8], non smooth solutions are penalised here and the minimization becomes

$$
\min _{N \geq 0}\|K N-R\|^{2}+\lambda\|D N\|^{2}
$$

where $D$ is the finite difference matrix chosen to approximate the integral of the second derivative of $N$ :

$$
D=\left(\begin{array}{cccccc}
-1 & 2 & -1 & 0 & \cdots & 0 \\
0 & \ddots & \ddots & \ddots & \ddots & \vdots \\
\vdots & \ddots & \ddots & \ddots & \ddots & 0 \\
0 & \cdots & 0 & -1 & 2 & -1
\end{array}\right)
$$

Phillips-Twomey method is used here: a regularization parameter $\lambda$ is chosen to calculate the corresponding regularization matrix $\tilde{K}, \tilde{K}=K^{t} K+\lambda D^{t} D$ and then minimization of $\|\tilde{K} N-R\|^{2}$ using Non-Negative-Least-Squares developed by Lawson [12] is performed. The regularization hyperparameter is chosen with a graphical technique using the L-curve criterion [13]. Indeed, data are noisy and we choose not to give any prior knowledge on the input error structure, so this technique seems convenient. At this point, model of measurement and inversion have been developed. A question that naturally rises from here is how perturbations in the input space parameters affect the reconstruction of the size distribution. Figure 3 illustrates the necessity of sensitivity analysis for the all set of parameters.

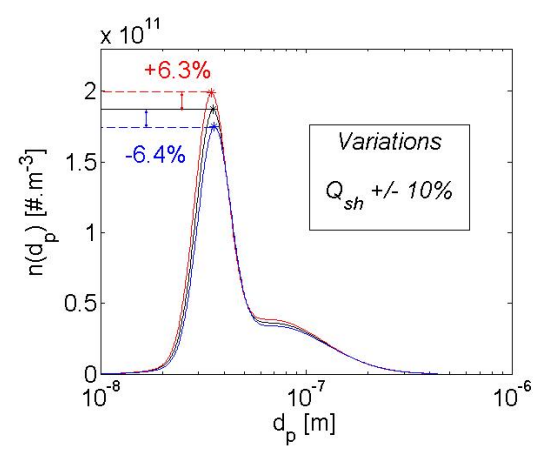

Fig. 3. Sensitivity of the estimate of the aerosol size distribution when flow rate $Q_{s h}$ is varied from $\pm 10 \%$. The mode value is estimated with an absolute error around $6 \%$. 


\section{UNCERTAINTY ANALYSIS}

In this part, a first uncertainty analysis is carried out to determine a confidence region on a simulated aerosol size distribution. Let's consider $\mathcal{M}: R^{d} \mapsto R^{J}$ the modelling function such that:

$$
\hat{N}=\mathcal{M}(\theta, R),
$$

where $\hat{N} \in R^{J}$ is the estimated aerosol size distribution vector and $\theta=\left(\theta_{1}, \theta_{2}, \ldots, \theta_{d}\right)$ is the $d$-dimensional real vector of input parameters (geometrical parameters, environmental parameters, physical parameters, etc.) that is then decomposed in two sets of parameters: uncertain parameters denoted as vector $\theta_{u}$ and fixed parameters with vector $\theta_{f}$. So, $\theta$ becomes $\theta=\left(\theta_{f}, \theta_{u}\right)$ with $\theta_{f}=\left(\theta_{f, 1}, \ldots, \theta_{f, d_{f}}\right) \in R^{d_{f}}$ and $\theta_{u}=\left(\theta_{u, 1}, \ldots, \theta_{u, d_{u}}\right) \in R^{d_{u}}$, $d=d_{u}+d_{f}$. As a first approach, uniform probability distributions are assigned to uncertain parameters $\theta_{u}$ to model their associated uncertainty as follows:

$$
\theta_{u, i} \sim \mathcal{U}\left(\left[a_{i}, b_{i}\right]\right) \quad, i=1, \ldots, d_{u} .
$$

However, the number of input parameters is high $(d \sim 40)$, so comes the priority to reduce the problem's dimension. It can be done with global sensitivity analysis methods. Since $n\left(d_{p}\right)$ is not a scalar but a function of the mobility diameter, we pick some convenient functional as peaks width or peaks position. They play the role of surrogate measurements used for the global sensitivity analysis. According to the screening method called OAT Morris method [14], parameters that have been found to impact the most the reconstruction of the size distribution are denoted as the vector $\theta_{u}^{m}, \theta_{u}^{m}=\left(Q_{s h}, Q_{a}, Q_{c p c}, Q_{e x c}, r_{1}, r_{2}, L_{D M A}, \Delta_{t}, \gamma\right)$. Since the dimension of the problem has been significantly reduced, Monte Carlo simulations can now be performed. Now that significant parameters have been discriminated, others are fixed. Let $\theta_{u}^{f}$ these part of uncertain parameters considered as fixed, then new vector $\theta_{u}$ can be written as $\theta_{u}=\left(\theta_{u}^{f}, \theta_{u}^{m}\right)$. If exponent ${ }^{[k]}$ denotes the $k$-th Monte Carlo simulation, then

$$
\hat{N}^{[k]}=\mathcal{M}\left(\theta_{f},\left(\theta_{u}^{f}, \theta_{u}^{m,[k]}\right), R\right) .
$$

Figure 4 represents a $95 \%$ confidence interval based on uncertainty propagation results when an atmospheric aerosol is simulated. According to our knowledge, this is the first time that a confidence region is associated to an aerosol size distribution. Monte Carlo simulations have become possible in this case thanks to requirements expressed in terms of modeling and inversion.

\section{Discussion}

A SMPS measurement model and associated uncertainties has been presented. They allow SMPS users to check their inversion method and also provide them with an idea of how uncertain the estimation of the aerosol size distribution can be. The uncertainty propagation is performed through the full model (measurement and inversion). However, In order to answer more precisely to SMPS users'

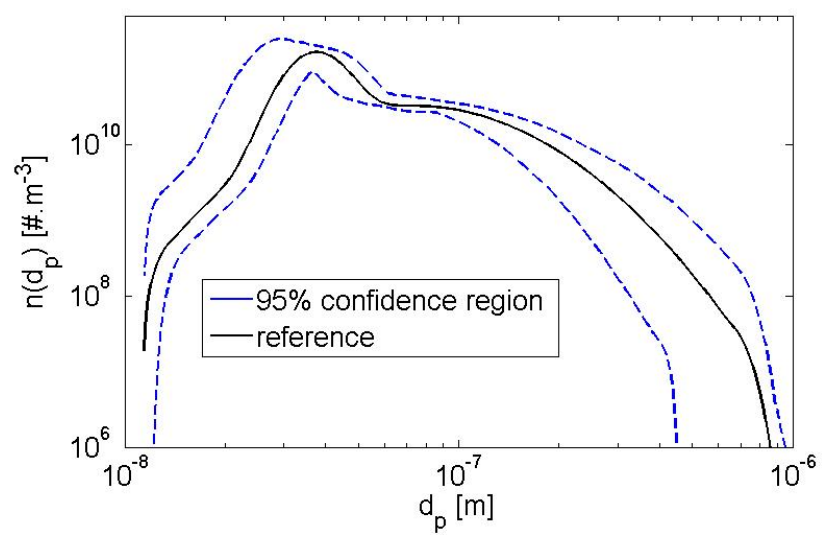

Fig. 4. 95\% Confidence region on an atmospheric aerosol size distribution

need, the uncertainty analysis could be rethought as a decomposition of the global model into two sub-models. Moreover, improvements have to be brought concerning the probability distributions assigned to every uncertain parameter in order to get closer to the physical reality. Following this statement, a counting noise will also be added to the raw data response function.

\section{REFERENCES}

[1] F. R. C. Wang, S. C., "Scanning electrical mobility spectrometer," Aerosol Science and Technology, vol. 13, pp. 230-240, 1990.

[2] C. D. R. F. R. C. S. J. H. Collins, D. R., "The scanning dma transfer function," Aerosol Science and Technology, no. 38, pp. 833-850, 2004.

[3] A. Wiedensohler, "An approximation of the bipolar charge distribution for particles in the submicron size range," Journal of Aerosol Science, vol. 19, pp. 387-389, 1988.

[4] R. Gunn, "Diffusion charging of atmospheric droplets by ions, and the resulting combination pp. 339-347, 1954.

[5] F. R. Quant, "Performance of condensation particle counters with three continuous-flow designs," Journal of Aerosol Science, vol. 23, pp. 405-408, 1992.

[6] M. G. K. S. P. D. Kim, J.H, "Slip correction measurements of certified psl nanoparticles using a nanometer differential mobility analyser (nano-dma) for knudsen number from 0.5 to 83," Journal of Research of National Institute of Standards and Technology, vol. 110, pp. 31-54, 2005.

[7] W. K. T. Knutson, E.O, "Accurate measurement of aerosol electric mobility moments," Journal of aerosol science, vol. 6, pp. 453-460, 1975.

[8] W. J. Seinfeld, J.H., "Inversion aerosol size distribution data," Journal of Aerosol Science, vol. 21, no. 2, pp. 227-247, 1990.

[9] A. D. J. Hagen, D. E., "Linear inversion method to obtain aerosol size distributions from measurements with a differential mobility analyser," Aerosol Science and Technology, vol. 4, no. 2 , pp. $465-475,1983$.

[10] S. Twomey, "Comparison of constrained linear inversion and an iterative non-linear algorithm applied to the indirect estimation of particle size distributions," Journal of Computational Physics, vol. 18, pp. 188-200, 1975.

[11] A. V. Y. Tikhonov, A. N., "Solutions of ill-posed problems," 1977.

[12] H. R. Lawson, C. L., "Solving least squares problems," pp. 345349, 1974.

[13] P. Hansen, "Analysis of discrete ill-posed problems by means of the l-curve," SIAM Rev, vol. 34, no. 4, p. 561?580, 1999.

[14] M. D. Morris, "Factorial sampling plans for preliminary computational experiments," Technometrics, vol. 33, p. 161?174, 1991. 